\title{
Cytopenia among CML Patients on Imatinib in Kenya: Types, Grades, and Time Course
}

\author{
Angela McLigeyo ${ }^{D},{ }^{1}$ Jamilla Rajab, ${ }^{2}$ Mohammed Ezzi, ${ }^{2}$ Peter Oyiro, ${ }^{3}$ Yatich Bett, ${ }^{4}$ \\ Andrew Odhiambo, ${ }^{2}$ Matilda Ong'ondi, ${ }^{3}$ Sitna Mwanzi $\mathbb{D},{ }^{5}$ Mercy Gatua, ${ }^{2}$ \\ and NAOthieno- Abinya ${ }^{2}$ \\ ${ }^{1}$ Department of Medicine, Maseno University, Kisumu, Kenya \\ ${ }^{2}$ Department of Clinical Medicine and Therapeutics, University of Nairobi, Nairobi, Kenya \\ ${ }^{3}$ Hemato-Oncology Unit, Kenyatta National Hospital, Nairobi, Kenya \\ ${ }^{4}$ Moi Teaching and Referral Hospital, Eldoret, Kenya \\ ${ }^{5}$ Aga Khan University Teaching Hospital, Nairobi, Kenya
}

Correspondence should be addressed to Angela McLigeyo; awinoligeyo@gmail.com

Received 22 June 2019; Revised 31 March 2020; Accepted 25 April 2020; Published 12 May 2020

Academic Editor: David H. Vesole

Copyright (c) 2020 Angela McLigeyo et al. This is an open access article distributed under the Creative Commons Attribution License, which permits unrestricted use, distribution, and reproduction in any medium, provided the original work is properly cited.

\begin{abstract}
Background. Imatinib mesylate is the gold standard for the treatment of all phases of Philadelphia-positive chronic myeloid leukemia. Patients on imatinib treatment may develop cytopenia due to drug toxicity. This study aimed to determine the types, grades, and time course of cytopenia in CML patients on imatinib at a Nairobi hospital. Methods. This was a cross-sectional descriptive study of adult patients aged $\geq 18$ years followed up at the Glivec International Patient Access Program (GIPAP) clinic from 2007 to 2015. Patients who developed cytopenia within 12 months of initiating imatinib were eligible. Clinical and hematologic data were retrieved from the patients' charts and entered into a study proforma. Measures of central tendency such as mean, median, mode, standard deviation, and variance were used for analysis. Results. Sixty three percent (63.6\%) of the 94 patients developed a monocytopenia, with anemia seen in 34\%, neutropenia in $27.6 \%$, and thrombocytopenia in $8 \%$ of the 94 patients. Anemia plus neutropenia was the most common bicytopenia at $12.7 \%$. Pancytopenia was seen in only 5 of the 94 patients. Most of the cytopenia was grades 2 and 3. Anemia was present at baseline while neutropenia and thrombocytopenia developed within 12 months of imatinib initiation. Anemia resolved during the first 12 months of therapy while neutropenia and thrombocytopenia resolved within 24-36 months of treatment. Conclusion. Monocytopenia, especially anemia, was the most common type of cytopenia. The cytopenia was predominantly grade 2, developed in majority of the patients within 6 months after imatinib initiation, and had resolved by $24-36$ months after imatinib initiation.
\end{abstract}

\section{Introduction}

Chronic myeloid leukemia (CML) is due to a clonal disorder that causes granulocyte cell line proliferation [1]. It develops following a translocation that occurs reciprocally between two somatic chromosomes, $t$ (9:22) [2]. The fusion protein resulting from this translocation, the BCR-ABL1, is a tyrosine kinase which acts independently of any stimulation [3]. Tyrosine kinase inhibitors (TKI) used in the treatment of CML block this kinase which in turn block signaling pathways involved in proliferation while stimulating apoptosis and cellular adhesion $[4,5]$. Patients on imatinib, a TKI, have been reported to develop cytopenia during the course of treatment. Sneed, in their study of $143 \mathrm{CML}$ patients on imatinib, reported that neutropenia and thrombocytopenia $\geq$ grade 3 developed in $64(45 \%)$ and 31 (22\%) patients, respectively [6]. A study conducted in Hyderabad among 683 CML patients aged between 21 and 75 years treated with imatinib reported that 46,25 , and 37 patients developed grade 2 anemia, neutropenia, and 
thrombocytopenia, respectively. Among them, 18 and 13 were reported as bicytopenia and pancytopenia, respectively [7]. The cytopenia was mild with majority of the patients having grade 1 or 2 toxicity [7].

Most of the hematologic toxicities develop but also resolve early following the initiation of imatinib [8] and are potentially reversible with either dose reduction or temporary imatinib discontinuation. Severe hematologic toxicity may occur with higher doses of imatinib used in the setting of imatinib resistance [9]. In the IRIS trial, the development of new onset anemia, thrombocytopenia, and neutropenia after 5 years of follow-up was rare at $4 \%, 9 \%$, and $17 \%$, respectively, and diminished over time during follow-up. In addition, grade 3 or 4 myelosuppression was infrequent after the initial two years of therapy [10].

In the GIPAP clinic in Nairobi, several patients have been reported to develop cytopenia immediately after imatinib initiation. These patients are managed with either a dose reduction or a treatment interruption. This study aimed to describe the type, grade, and time course of cytopenia among patients treated with imatinib. It will add to the scientific knowledge on types and grades of cytopenia and aid in decision making for these patients.

\section{Methods}

2.1. Study Setting. The Max access program provides free imatinib to patients in Kenya at the GIPAP clinics. $\mathrm{Cu}$ mulatively, the clinic at the Nairobi hospital, Kenya, has enrolled $1200 \mathrm{CML}$ patients. An average of 150 patients attend the clinic bi-weekly. The clinic is centralized and receives patients from the entire country. The age range of patients seen in the clinic is 6 to 75 years. The males that attend the clinic are in similar proportion to the females, and almost $90 \%$ are in the chronic phase of CML. Patients who initiate treatment are compliant with treatment with adherence rates of approximately 80\% [11].

2.2. Study Design and Population. This was a cross-sectional descriptive study of 94 patients. CML patients aged $\geq 18$ years attending GIPAP clinic from 2007 to 2015 and on imatinib $400 \mathrm{mg}$ daily who developed cytopenia $\geq$ grade 2 were enrolled.

2.3. Data Collection. Data collection was conducted over a 3month period. Access to the files was limited to the principal investigator (PI) and a trained study assistant. A coded questionnaire was used as the study instrument to abstract the information from the files. The principal investigator reviewed the data to ensure completeness and accuracy. The patient's names were left out, and instead, each patient was assigned a numerical identifier. Data were extracted from 2007 to 2015.

2.4. Data Management and Statistical Analysis. Data were transferred into Microsoft Excel and imported into the statistical analysis software for data management and analysis. Continuous data were presented using means and respective standard deviations (SD). Counts and corresponding percentages were used for categorical variables. Stata package, version 15.1, was used during statistical analysis. Statistical tests were evaluated for significance at the $5 \%$ level $(p<0.05)$. Tables, bar charts, pie charts, and line graphs were used to display results.

2.5. Variables. Cytopenia was determined from the complete blood count (CBC) report of hemoglobin, neutrophil, and platelet counts. Monocytopenia was defined as an abnormality in one parameter, bicytopenia as an abnormality in two parameters, and pancytopenia as an abnormality in three parameters based. Categorization of severity was based on the National Cancer Institute Common Terminology Criteria for Adverse Events v.3 (NCI CTAE v3) [12].

Time to development of cytopenia after initiation of imatinib was categorized as less than 3 months, 3-6 months, and 6-12 months while duration of cytopenia was determined from annual CBC reports over a 36-month period.

2.6. Ethical Considerations. Ethical approval was obtained from the KNH/UON Ethics and Research Committee. Data were stored in a password-protected computer. The study was a minimal risk study since there was no direct patient involvement. For confidentiality, the patients' charts were used only within the confines of the records department of the clinic.

\section{Results}

3.1. Type of Cytopenia. Sixty-six patients (63.6\%) among the 94 studied had a monocytopenia. Anemia developed in 32 (34\%), neutropenia in $26(27.6 \%)$, and thrombocytopenia in $8(8 \%)$ of the 94 patients. Among patients with bicytopenia, the most common type was anemia plus neutropenia in 12 $(12.7 \%)$ patients, followed by neutropenia plus thrombocytopenia in $8(8 \%)$ patients and anemia and thrombocytopenia in $3(3 \%)$ patients. Pancytopenia developed in 5 patients among the 94 studied.

3.2. Grade of Cytopenia. Grade 2 and 3 cytopenia were equally common at $48.5 \%$ and $43.9 \%$, respectively, as shown in Table 1.

3.3. Time to Development of Cytopenia. Thirty-seven (40\%) patients developed cytopenia within 3 months of initiating imatinib, 33 (34.7\%) within 3-6 months, and $24(25.3 \%)$ within 6-12 months.

3.4. Duration of Cytopenia. All grades of anemia improved from the reported baseline and resolved within 12 months (Figure 1). Thrombocytopenia improved from 12 months and had resolved by 36 months except in one patient (Figure 2). Neutropenia also began to improve after 12 months for the affected patients and had resolved by 24-36 months (Figure 3). 
TABLE 1: Grades of cytopenia among the 66 patients with monocytopenia, NCI CTCAE, v3.

\begin{tabular}{lccc}
\hline Variables & Grade 2 & Grade 3 & Grade 4 \\
\hline Anemia $(\mathrm{g} / \mathrm{dL}), n(\%)$ & $8-10$ & $6.5-8$ & $<6.5$ \\
& $17(53)$ & $13(40.6)$ & $2(6.4)$ \\
Neutropenia $\left(\mathrm{mm}^{3}\right), n$ & $\geq 1000-1500$ & $500-1000$ & $<500$ \\
$(\%)$ & $10(38.5)$ & $14(53.8)$ & $2(7.7)$ \\
Thrombocytopenia & $50000-75000$ & $25000-50000$ & $<25000$ \\
$\left(\mathrm{~mm}^{3}\right), n(\%)$ & $5(62.5)$ & $2(25)$ & $1(12.5)$ \\
Total, $n(\%)$ & $32(48.5)$ & $29(43.9)$ & $5(7.6)$ \\
\hline
\end{tabular}

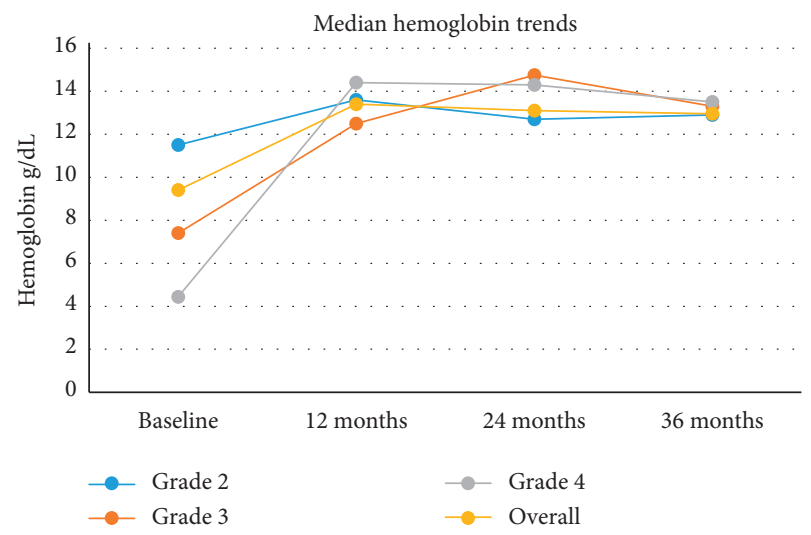

Figure 1: Median hemoglobin trends over a 36-month time period.

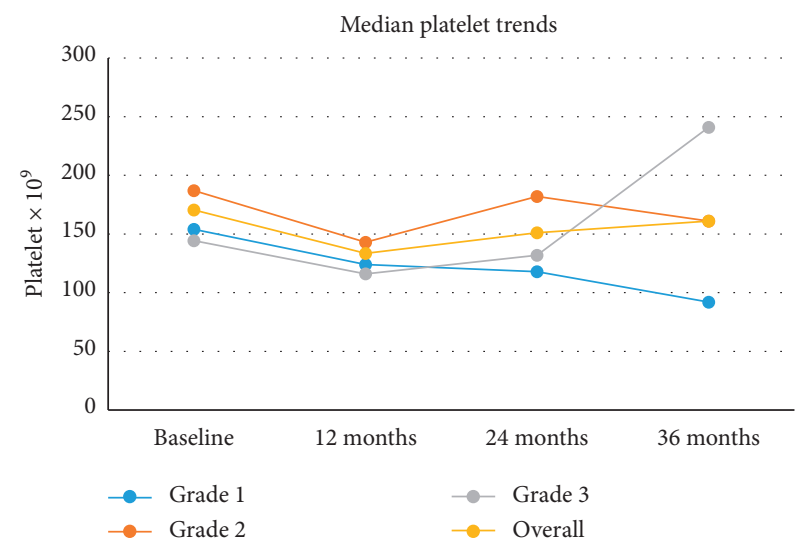

Figure 2: Median platelet values over a 36-month time period.

\section{Discussion}

The study was conducted among 94 patients with cytopenia. More patients developed monocytopenia (71\%) than either bicytopenia $(24 \%)$ or pancytopenia $(5 \%)$. Anemia was the most common monocytopenia, and anemia plus neutropenia was the most common bicytopenia. Pancytopenia was seen in only 5 of the 94 patients. Grade 2 and 3 cytopenia was common at $48.5 \%$ and $43.9 \%$, respectively. Majority of the cytopenia (74\%) developed within six months of initiating imatinib and resolved within 12 months for anemia

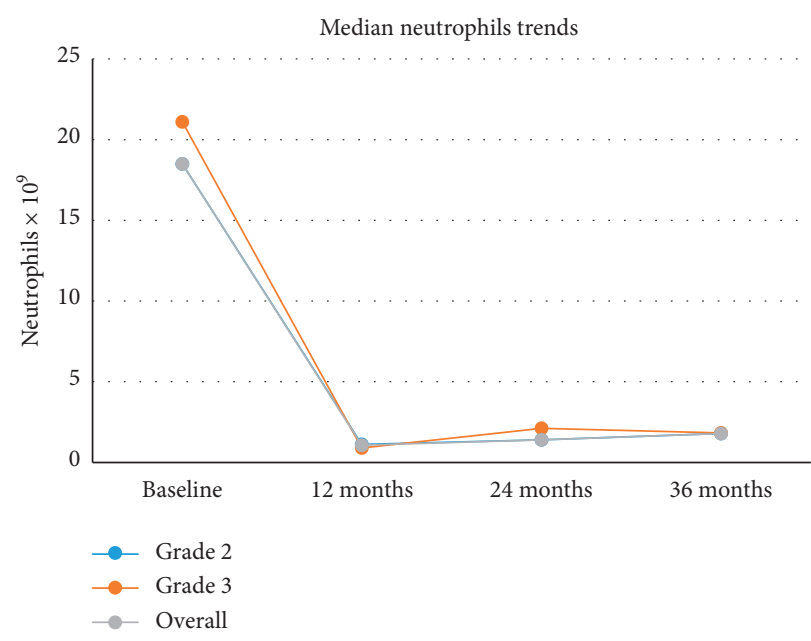

FIGURE 3: Median neutrophil values over a 36-month time period.

and within 24-36 months for thrombocytopenia and neutropenia. Our data are similar to that from India where anemia developed in 46 (35\%), thrombocytopenia in 34 (25\%), neutropenia in 24 patients (17\%), and bicytopenia in 18 patients. This study included grade 2 to 4 cytopenia in the analysis and was conducted in a low-resource setting similar to ours [7]. Similarly, a different study from India reported that anemia was the most frequent myelotoxicity and occurred in $129(65 \%)$ participants, followed by neutropenia in $57(28 \%)$ and thrombocytopenia in 34 (17\%) participants [13]. In another trial, during the first 12 months of treatment, grade 3 and 4 toxicity was noted among the 532 study participants. Neutropenia developed in $33 \%$, thrombocytopenia in $18 \%$, and anemia in $6 \%$ of the patients [8]. In contrast, a study conducted in Indonesia reported lower levels of cytopenia with anemia at $20 \%$, thrombocytopenia at $14 \%$, and neutropenia at $4 \%$ [14] while a study from Iraqi documented anemia at $14 \%$, and neutropenia and thrombocytopenia at $10 \%$ and $4 \%$ [15]. Lower proportions of cytopenia reported in some studies may be due to the analysis of patients with grade 3 and 4 cytopenia only. Earlier diagnosis of CML in the developed world may also result in lower levels of hematological toxicity.

Our study reported grade 2 and 3 cytopenia as the most common grade. Similarly, an Indian study reported high proportions of grade 2 cytopenia at $18.5 \%, 8.8 \%$, and $10.3 \%$ for anemia, neutropenia, and thrombocytopenia, respectively, followed by grade 3 at $11.8 \%, 6.6 \%$, and $7.4 \%$ for anemia, neutropenia, and thrombocytopenia, respectively [7]. Druker et al. reported lower levels of severe cytopenia following imatinib therapy with grade 3-4 neutropenia at $14-19 \%$, thrombocytopenia at $8-10 \%$, and anemia at $3-4 \%$ [10]. The IRIS trial likewise reported severe cytopenia of grade 3 or 4 to be low with anemia at $3 \%$, neutropenia at $14.3 \%$, and thrombocytopenia at $8 \%$ [16]. In contrast, a study from Spain reported grade 3-4 cytopenia as being the predominant grade [17], and Zhou et al. reported grade 3 to 4 neutropenia, anemia, and thrombocytopenia in $21.8 \%$, $17.8 \%$, and $5.9 \%$ of patients with CML, respectively [18]. The presence of grade 3-4 toxicity may be due to presentation 
with advanced CML. Kantarjian et al. reported higher grades of cytopenia in patients presenting with advanced chronic phase-CML, with $35 \%, 20 \%$, and $7 \%$ developing neutropenia, thrombocytopenia, and anemia, respectively, of grades 3 to 4 [19] while a similar study also reported that advanced CML and high imatinib doses of $600 \mathrm{mg}$ were associated with higher proportion of cytopenia [20].

Cytopenia developed within 3 months of imatinib initiation in 40\%, 3-6 months in 34.7\%, and 6-12 months in $25 \%$ of the patients. Myelosuppression may develop at any time during treatment of CML but is common in the initial 2-4 weeks of therapy, especially for advanced disease [21]. Other studies have reported onset of cytopenia between 3 and 5 weeks of imatinib therapy [22].

The development of severe cytopenia rapidly in patients with a myeloid bulge shortly after TKI initiation is a phenomenon that is still under study. The elevation of leucocytes in these patients probably reflects the high disease burden at diagnosis [19]. Once normal blood cell formation resumes, the myelosuppression that has been induced by TKI treatment resolves and the risk of developing myelosuppression later in the course of treatment reduces. In addition, the blood and marrow laboratory results often normalize in the course of follow-up [8]. This was reported in our study where there was recovery of anemia, thrombocytopenia, and neutropenia at the end of the 36-month follow-up. In other studies, grade 3 and grade 4 cytopenia resolved soon after treatment interruption and did not recur with resumption of treatment [23]. The IRIS trial similarly reported that myelosuppression developed early in the treatment course with imatinib therapy but had resolved in the majority of patients after the first 12 months of follow-up [24]. The recovery of the cell counts within 12 months of initiating treatment could reflect bone marrow normalization and regeneration [25]. However, some isolated cases of recurrent myelosuppression requiring extended treatment interruptions have been reported and may be a consequence of advanced disease [17]. Conversely, the cytopenia may recover within days after its development as has been documented prior where the median duration of thrombocytopenia and neutropenia was less than 3 weeks [23]. The duration of cytopenia may also be influenced by duration of CML prior to diagnosis. A study reported that patients with long duration of CML disease prior to diagnosis tended to have more advanced disease. In the event of myelotoxicity in these patients, the duration of cytopenia was more prolonged, especially with higher grades of cytopenia [26].

In conclusion, monocytopenia was common, was of grades 2 and 3, developed early in the course of treatment, and resolved relatively early during follow-up. Clinicians involved in the management of these patients should be alert to diagnose, manage, and monitor these patients.

\section{Data Availability}

The datasets used and/or analyzed during the current study are available from the corresponding author on reasonable request.

\section{Disclosure}

This manuscript is based on a thesis by the authors. The full thesis can be assessed at http://erepository.uonbi.ac.ke/ handle/11295/105816.

\section{Conflicts of Interest}

The authors declare that there are no conflicts of interest regarding the publication of this paper.

\section{References}

[1] M. Pathophysiology and C. Features, Chronic Myeloid Leukemia, Springer, Berlin, Germany, 2016.

[2] J. M. Goldman and J. V. Melo, "Chronic myeloid leukemia-advances in biology and new approaches to treatment," New England Journal of Medicine, vol. 349, no. 15, pp. 1451-1464, 2003.

[3] A. Quintás-Cardama and J. Cortes, "Molecular biology of bcrabll-positive chronic myeloid leukemia," Blood, vol. 113, no. 8, pp. 1619-1630, 2009.

[4] M. W. Deininger, S. Vieira, R. Mendiola, B. Schultheis, J. M. Goldman, and J. V. Melo, "BCR-ABL tyrosine kinase activity regulates the expression of multiple genes implicated in the pathogenesis of chronic myeloid leukemia," Cancer Research, vol. 60, no. 7, pp. 2049-2055, 2000.

[5] Z. Aziz, J. Iqbal, M. Akram, and S. Saeed, "Treatment of chronic myeloid leukemia in the imatinib era," Cancer, vol. 109, no. 6, pp. 1138-1145, 2007.

[6] T. B. Sneed, H. M. Kantarjian, M. Talpaz et al., "The significance of myelosuppression during therapy with imatinib mesylate in patients with chronic myelogenous leukemia in chronic phase," Cancer, vol. 100, no. 1, pp. 116-121, 2004.

[7] T. R. Paul and S. G. Uppin, "Evaluation of cytopenias occurring in imatinib treated chronic myeloid leukemia ( CML ) patients," Indian Journal of Hematology and Blood Transfusion, vol. 26, no. 2, pp. 56-61, 2010.

[8] A. Hochhaus, B. Druker, C. Sawyers et al., "Favorable longterm follow-up results over 6 years for response, survival, and safety with imatinib mesylate therapy in chronic-phase chronic myeloid leukemia after failure of interferon- $\alpha$ treatment," Blood, vol. 111, no. 3, pp. 1039-1043, 2008.

[9] B. Stein and B. Douglas Smith, "Treatment options for patients with chronic myeloid leukemia who are resistant to or unable to tolerate imatinib," Clinical Therapeutics, vol. 32, no. 5, pp. 804-820, 2010.

[10] B. J. Druker, F. Guilhot, S. G. O'Brien et al., "Five-year followup of patients receiving imatinib for chronic myeloid leukemia," New England Journal of Medicine, vol. 355, no. 23, pp. 2408-2417, 2006.

[11] G. W. Kiarie, N. A. Othieno-Abinya, and M. S. Riyat, "The GLIVEC international patient assistance programme: the Nairobi experience," East African Medical Journal, vol. 86, no. 12, pp. 106-107, 2009.

[12] Cancer Therapy Evaluation Program, "Common Terminology Criteria for Adverse Events, Version 3.0, DCTD, NCI, NIH, DHHS," 2003, http://ctep.cancer.gov.

[13] S. Rajappa, L. Varadpande, T. Paul, R. Jacob, and R. Digumarti, "Imatinib mesylate in early chronic phase chronic myeloid leukemia: experience from a developing country," Leukemia \& Lymphoma, vol. 49, no. 3, pp. 554-558, 2008. 
[14] A. H. Reksodiputro, S. Syafei, N. Prayogo et al., "Clinical characteristics and hematologic responses to Imatinib in patients with chronic phase myeloid leukemia (CML) at Cipto Mangunkusumo Hospital," Acta Medica Indonesiana, vol. 42, no. 1, pp. 2-5, 2010.

[15] B. F. Matti, A. S. Naji, and A. F. Alwan, "Evaluation of the safety of imatinib mesylate in 200 Iraqi patients with chronic myeloid leukemia in the chronic phase: single-center study," The Turkish Journal of Hematology, vol. 30, no. 4, pp. 38739393, 2013.

[16] S. G. O'Brien, F. Guilhot, R. A. Larson et al., "Imatinib compared with interferon and low-dose cytarabine for newly diagnosed chronic-phase chronic myeloid leukemia," New England Journal of Medicine, vol. 348, no. 11, pp. 994-1004, 2003.

[17] F. Cervantes, J. Hernández-Boluda, J.-L. Steegmann et al., "Imatinib mesylate therapy of chronic phase chronic myeloid leukemia resistant or intolerant to interferon: results and prognostic factors for response and progression-free survival in 150 patients," Haematologica, vol. 88, pp. 1117-1122, 2003.

[18] Y. Zhou and S. X. Qian, "Clinical efficacy and safety of imatinib in the management of $\mathrm{Ph}+$ chronic myeloid or acute lymphoblastic leukemia in Chinese patients," OncoTargets and Therapy, vol. 7, pp. 395-404, 2014.

[19] H. M. Kantarjian, J. E. Cortes, S. O’Brien et al., "Imatinib mesylate therapy in newly diagnosed patients with Philadelphia chromosome-positive chronic myelogenous leukemia: high incidence of early complete and major cytogenetic responses," Blood, vol. 101, no. 1, pp. 97-100, 2003.

[20] M. Talpaz, R. T. Silver, B. J. Druker et al., "Imatinib induces durable hematologic and cytogenetic responses in patients with accelerated phase chronic myeloid leukemia: results of a phase 2 study Imatinib induces durable hematologic and cytogenetic responses in patients with accelerated phase chron," Trials, vol. 99, no. 6, pp. 1928-1937, 2009.

[21] F. Guilhot, "Indications for imatinib mesylate therapy and clinical management," The Oncologist, vol. 9, no. 3, pp. 271-281, 2004.

[22] A. Barber, W. Afzal and M. Akhtari, Hematologic toxicities of small molecule tyrosine kinase inhibitors." Targeted oncology, vol. 6, pp. 203-215, 2011.

[23] I. Cojbasic and L. Macukanovic-Golubovic, "Hematopathologic and cytogenetic findings in imatinib mesylate treated chronic myelogenous leukemia patients: 2.5 years' experience," Vojnosanitetski Pregled, vol. 67, no. 10, pp. 802-806, 2010.

[24] B. J. Druker, F. Guilhot, S. O'Brien, and R. A. Larson, "Longterm benefits of imatinib (IM) for patients newly diagnosed with chronic myelogenous leukemia in chronic phase (CML$\mathrm{CP}$ ): the 5-year update from the IRIS study," Journal of Clinical Oncology, vol. 24, no. 18_suppl, p. 6506, 2006.

[25] N. C. Narang, "Morphological changes in bone marrow post imatinib therapy in chronic phase CML: a follow up study on sequential bone marrow aspirates and biopsies," Journal of Clinical and Diagnostic Research, vol. 11, no. 4, pp. 25-29, 2017.

[26] H. Kantarjian, R. Pasquini, N. Hamerschlak et al., "Dasatinib or high-dose imatinib for chronic-phase chronic myeloid leukemia after failure of first-line imatinib: a randomized phase 2 trial," Blood, vol. 109, no. 12, pp. 5143-5150, 2007. 